\title{
Prevalence and Antimicrobial Resistance of Salmonella Isolated from Animal-Origin Food Items in Gondar, Ethiopia
}

\author{
Mebrat Ejo, ${ }^{1}$ Legesse Garedew, ${ }^{2}$ Zabishwork Alebachew, ${ }^{3,4}$ and Walelgn Worku ${ }^{4}$ \\ ${ }^{1}$ Department of Biomedical Sciences, Faculty of Veterinary Medicine, University of Gondar, Gondar, Ethiopia \\ ${ }^{2}$ Department of Microbiology, St. Paul's Hospital Millennium Medical College, Addis Ababa, Ethiopia \\ ${ }^{3}$ Department of Veterinary Epidemiology and Public Health, Faculty of Veterinary Medicine, University of Gondar, Gondar, Ethiopia \\ ${ }^{4}$ Institute of Public Health, College of Medicine and Health Sciences, University of Gondar, Gondar, Ethiopia
}

Correspondence should be addressed to Mebrat Ejo; drmebrat@yahoo.com

Received 12 July 2016; Revised 18 November 2016; Accepted 23 November 2016

Academic Editor: Ernesto S. Nakayasu

Copyright (c) 2016 Mebrat Ejo et al. This is an open access article distributed under the Creative Commons Attribution License, which permits unrestricted use, distribution, and reproduction in any medium, provided the original work is properly cited.

\begin{abstract}
Salmonella has been found to be the major cause of foodborne diseases and a serious public health problem in the world, with an increasing concern for the emergence and spread of antimicrobial-resistant strains. A cross-sectional study was conducted between February 2014 and December 2015 on food items of animal origin to assess the prevalence and antimicrobial resistance profiles of Salmonella isolates using standard bacteriological methods. The overall prevalence rate of $5.5 \%$ was recorded from the total analyzed food items of animal origin. Salmonella isolates were detected from $12 \%$ of raw meat, $8 \%$ of minced meat, $2.9 \%$ of burger samples, $18 \%$ of raw eggs, and $6 \%$ of raw milk. Furthermore, antimicrobial susceptibility test identified $47.6 \%$ resistant Salmonella isolates, $28.6 \%$ intermediately sensitive isolates, and 23.8\% susceptible isolates. Among Salmonella isolates tested, $42.6 \%, 28.6 \%$, and $14.3 \%$ were found to be relatively resistant to tetracycline, sulfamethoxazole-trimethoprim, and ampicillin, respectively, while $9.5 \%-$ $19 \%$ were intermediately resistant to tetracycline, amoxicillin, ampicillin, cephalothin, and nitrofurantoin. Therefore, our findings provide the prevalence and drug resistance of Salmonella from foods of animal origin and contribute information to scientists as well as public health researchers to minimize the prevalent and resistant foodborne Salmonella species in Ethiopia.
\end{abstract}

\section{Background}

Foodborne diseases are among the most widespread global public health problems of recent times, and their implication for health and economy is increasingly recognized $[1,2]$. According to reports, every year, a huge number of people suffer from foodborne diseases worldwide due to contaminated food and water consumption $[2,3]$. A wide range of pathogens play a role in foodborne disease, most of which have a zoonotic origin and have carriers in healthy food animals from which they spread to an increasing variety of foods of animal origin and are considered as major vehicles of foodborne infections [4]. Among the pathogens, Salmonella is considered the most prevalent foodborne pathogen worldwide $[4,5]$ and has long been recognized as an important zoonotic microorganism of economic significance in animals and humans [5], predominantly in the developing countries. Consumption of raw or unsafe food, cross-contamination, improper food storage, poor personal hygiene practices, inadequate cooling and reheating of food items, and a prolonged time lapse between preparing and consuming food items were mentioned as contributing factors to an outbreak of salmonellosis in humans $[3,5]$. The ubiquity of Salmonella isolates creates a persistent contamination hazard in all raw foods [5] and also in animal-origin food products, which are often implicated in sporadic cases and outbreaks of human salmonellosis [2, 6]. Foodborne transmission is recognized as the major cause of Salmonella infections, with many food sources and supplies implicated in these infections [2].

Antibiotic-resistant Salmonella infections of both human and animal are universal concerns, particularly in developing countries where the risk of infection is high because of unhygienic living conditions, close contact and sharing of houses between animals and humans [7], and the traditions of consumption of raw or undercooked animal-origin food items. There is an increasing concern with this pathogen due to the 
emergence and spread of antibiotic-resistant and potentially more pathogenic strains. Antimicrobial-resistant Salmonella spp. have been isolated from different foods of animal origin around the world, which is attributed to the inappropriate use of antimicrobials as therapeutic or prophylactic agents in human and veterinary medicine, as well as the use of growth promoters in animal production [8]. Salmonella strains resistant to various antimicrobial agents, particularly resistant to fluoroquinolones and third-generation cephalosporins, are considered as an emerging problem worldwide [9], resulting in higher morbidity and mortality rates and higher overall treatment costs. This may represent a public health risk by transfer of resistant Salmonella strains to humans through the consumption of contaminated food and food products. However, the sources and transmission routes of Salmonella in developing countries are poorly understood due to the lack of coordinated national epidemiological surveillance systems [3].

The incidence of foodborne Salmonella infections has increased dramatically in Ethiopia during the past few years. Studies conducted in different parts of the country have demonstrated the presence of Salmonella in human beings $[6,10,11]$ and in different food animals and food products $[10,12-14]$. Despite these attempts to report the prevalence and distribution of Salmonella spp. in some food items, humans, and food animals in Ethiopia [10-12], the problem of this pathogen in foods of animal origin is still not well known. However, studies made elsewhere indicated that foods of animal origin are important sources of Salmonella, particularly among those raw food consumers. In Gondar, the most touristic city in Ethiopia, there is not enough information on foodborne Salmonella prevalence in different food items such as foods from animal origin or others. Therefore, this study reports the presence of Salmonella including its antimicrobial-resistant profiles in foods of animal origin in Gondar.

\section{Materials and Methods}

2.1. Study Location. The study was conducted in Gondar, which is located in the northwest of Ethiopia, $740 \mathrm{~km}$ away from the capital, Addis Ababa. The town is one of the historic and touristic centers in Ethiopia. The altitude of the town ranges from 1800 to 2200 meters above sea level. It has average rainfall of 1000 millimeters per annum. The annual average maximum and minimum temperature of the town are $30.7^{\circ} \mathrm{C}$ and $22^{\circ} \mathrm{C}$, respectively, with an overall average of $26^{\circ} \mathrm{C}$. The town has relative humidity varying from $60 \%$ to $70 \%$ during the rainy season and from $30 \%$ to $40 \%$ during the dry season. The human population of the town is estimated at 214000 inhabitants [15]. In the town, a number of people are involved in the public service business, including smallholder farming supplying food animals and animal products to the communities and the slaughterhouse.

2.2. Study Design and Sampling. A cross-sectional study was conducted between February 2014 and December 2015 to assess the prevalence of Salmonella isolates and their antibiotic-resistant profiles from foods of animal origin in
Gondar. Stratified random sampling from catering establishments and a list frame of public places were used as sample source according to the accessibility of animal-origin food items. First, we looked up the functional catering establishments registered in the city and then we stratified the public places into strata (cafeterias, hotels, restaurants, and pastry and retail shops) and used them as a sampling frame. Study foods of animal origin were selected during dining time using a simple random sampling method in each public place on proportional bases.

2.3. Animal-Origin Food Items. A total of 384 animal-origin food samples were purchased from restaurants, hotels, cafeterias, and pastry and retail shops, including egg sandwich, minced and raw meat, burger patties, cottage cheese, cream cake, and beef pizza. In addition, raw egg and pasteurized and raw milk were bought from supermarkets and retail shops. Some of the animal-origin foods may not be considered "ready to eat" in all countries, as they would define "ready to eat" as having been processed, such as via cooking to a specific temperature for food safety. However, in Ethiopia, eating raw animal-origin foods like minced and raw beef, as well as milk, is a common practice. Time of sampling was scheduled at the beginning of the serving period (breakfast, lunch, or dinner time). Sterile forceps and spoons were used to transfer the food items from the eating plate to sampling containers. The collected samples were properly identified by sample type, date of collection, and sources and immediately transported to the laboratory in an ice box with freeze packs under completely sterile conditions for microbiological analysis [16].

2.4. Isolation and Identification of Salmonella. Standard cultivation method recommended by the International Organization for Standardization [16] was carried out for isolation and identification of Salmonella. Briefly, $25 \mathrm{gm}$ of each food sample was weighed and homogenized in sterile stomacher bag (Stomacher 400R, Seward, England) with $225 \mathrm{~mL}$ of preenrichment buffered peptone water for $2 \mathrm{~min}$. The nonselective preenriched sample, from each solid or liquid food sample, was mixed thoroughly and incubated overnight at $37^{\circ} \mathrm{C}$. Following incubation, $1 \mathrm{~mL}$ of the preenrichment broth was transferred to every $10 \mathrm{~mL}$ of tetrathionate broth (MüllerKaufmann, Oxoid, England), for first selective enrichment of Salmonella growth while inhibiting other microorganisms, and incubated at $37^{\circ} \mathrm{C}$ for $18-24$ hours. In addition, $0.1 \mathrm{~mL}$ of preenrichment broth was added to $10 \mathrm{~mL}$ of Rappaport-Vassiliadis (Oxoid, England) broth for second selective enrichment and incubated at $41^{\circ} \mathrm{C}$ for $18-24$ hours. For isolation of Salmonella species, each suspected colony from selectively enriched medium was streaked onto selective xylose lysine deoxycholate (XLD) agar plates and incubated at $37^{\circ} \mathrm{C}$ for $18-24$ hours. Salmonella suspected colonies from XLD agar medium were plated onto nutrient agar (Oxoid, England) plates to use for biochemical confirmation.

2.5. Biochemical Confirmation of the Isolates. Each identified colony with typical Salmonella morphology was confirmed biochemically by inoculating into triple sugar iron (TSI) agar, methyl red-Voges-Proskauer (MR-VP) broth, indole 
TABLE 1: Salmonella prevalence from animal-origin food items.

\begin{tabular}{lcc}
\hline Sample type & Number of samples tested & Number of samples that tested positive (\%) \\
\hline Burger & 35 & $1(2.9)$ \\
Cottage cheese & 20 & $0(0)$ \\
Cream cake & 50 & $0(0)$ \\
Egg sandwich & 35 & $0(0)$ \\
Minced meat & 25 & $2(8)$ \\
Pasteurized milk & 45 & $0(0)$ \\
Pizza & 24 & $0(0)$ \\
Raw egg & 50 & $9(18)$ \\
Raw meat & 50 & $6(12)$ \\
Raw milk & 50 & $3(6)$ \\
\hline Total & 384 & $21(5.5)$ \\
\hline
\end{tabular}

test, Simmons' citrate agar, urea agar, lysine iron agar (LIA), and SIM medium and incubated at $37^{\circ} \mathrm{C}$ for $18-48$ hours and interpreted with the International Organization for Standardization guidelines [16].

2.6. Antimicrobial Susceptibility Testing. Phenotypic antimicrobial susceptibility testing on Mueller-Hinton agar (Oxoid) using the agar disc diffusion method [17] was conducted to determine the antibiotic-resistant profiles of each isolate. Briefly, isolates were grown in a shaking water bath at $37^{\circ} \mathrm{C}$ until obtaining a $0.5 \mathrm{McF}$ arland turbidity standard. The bacterial suspension was then spread over the entire surface of Mueller-Hinton agar plates, and antibiotic disks including amoxicillin (20 mg), ampicillin (10 mg), cephalothin (30 mg), ceftriaxone $(30 \mathrm{mg})$, gentamycin $(10 \mathrm{mg})$, nalidixic acid $(30 \mathrm{mg})$, nitrofurantoin $(30 \mathrm{mg})$, sulfamethoxazoletrimethoprim $(30 \mathrm{mg})$, and tetracycline $(30 \mathrm{mg})$ were applied on the surface of the medium and incubated at $37^{\circ} \mathrm{C}$ for $18-$ 24 hours. Following incubation, the diameters of the inhibition zone were measured in millimeters and interpreted in accordance with CLSI guidelines [17]. Strains were evaluated as susceptible, intermediate, and resistant. An isolate was defined as resistant if it was resistant to one or more of the antimicrobials. Escherichia coli ATCC 25922 was used as a quality control and standard strain.

2.7. Statistical Analysis. Statistical analysis was performed using SPSS Statistics 17 (SPSS Inc., USA). Descriptive statistics such as frequency, percentage, and/or proportion were applied to compute the collected data from different foods of animal origin and antimicrobial susceptibility test results.

2.8. Ethical Considerations. Institutional permission to perform this study was obtained from the research and the ethical review committee of University of Gondar, Ethiopia. In addition, the research supporting letter was obtained from Gondar city administration and health office, Ethiopia. Confidentiality was ensured through the use of codes in records.

\section{Result}

3.1. Prevalence. In this study, 21 (5.5\%) out of 384 samples were found to be positive for Salmonella spp. (Table 1) through standard culture technique. All these isolates obtained from positive samples were confirmed primarily with the help of Salmonella spp. growth and morphological characteristics on selective media and then with biochemical tests (Table S1 in Supplementary Material available online at http://dx.doi.org/10.1155/2016/4290506). Salmonella isolates were detected from $6(12 \%)$ of the raw meat samples, 2 $(8 \%)$ of the minced meat samples, $1(2.9 \%)$ of the burger samples, $9(18 \%)$ of the raw eggs, and $3(6 \%)$ of the raw milk samples. However, cottage cheese, cream cake, egg sandwich, pasteurized milk, and pizza test samples were Salmonella negative. Salmonella was recovered more frequently from raw meat and raw eggs than from minced meat and/or raw milk. Among the sample sources, samples examined from the butcher house and retail shops with the prevalence of $13 \%$ and $13.3 \%$, respectively, were the most contaminated compared with the others (data not shown).

3.2. Antimicrobial Resistance Profile. The overall proportion of antimicrobial susceptibility test and the results of antimicrobial resistance of Salmonella spp. isolated from raw meat, minced meat, raw eggs, and raw milk are given in Tables 2 and 3, respectively. Of the total 21 Salmonella isolates subjected to antimicrobial susceptibility test using nine different antimicrobials, a total of 10 (47.6\%) Salmonella isolates were found to be resistant to one or more than one (multidrug) of the antimicrobials, and 6 (28.6\%) isolates showed an intermediate sensitivity to one or more antimicrobials, and 5 (23.8\%) isolates were susceptible to all tested antimicrobials. The Salmonella isolate from the burger sample was susceptible to all tested antimicrobial agents. In relation to the total Salmonella isolates tested, 42.6\%, 28.6\%, and $14.3 \%$ were found to be relatively resistant to tetracycline, sulfamethoxazole-trimethoprim, and ampicillin, respectively, while $9.5 \%-19 \%$ were intermediately resistant to tetracycline, amoxicillin, ampicillin, cephalothin, and 
TABLE 2: The general proportion of antimicrobial susceptibility test among Salmonella isolates.

\begin{tabular}{|c|c|c|c|}
\hline \multirow{2}{*}{ Antimicrobials } & \multicolumn{3}{|c|}{ Antimicrobial susceptibility test } \\
\hline & Susceptible (\%) & Intermediate (\%) & Resistant (\%) \\
\hline Amoxicillin (AML) & $16(76.2)$ & $3(14.3)$ & $2(9.5)$ \\
\hline Ampicillin (AMP) & $15(71.4)$ & $3(14.3)$ & $3(14.3)$ \\
\hline Cephalothin (KF) & $16(76.2)$ & $4(19)$ & $1(4.8)$ \\
\hline Ceftriaxone (CRO) & $21(100)$ & 0 & 0 \\
\hline Gentamicin $(\mathrm{CN})$ & $19(90.5)$ & 0 & $2(9.5)$ \\
\hline Nalidixic acid (NA) & $19(90.5)$ & 0 & $2(9.5)$ \\
\hline Nitrofurantoin (F) & $15(71.4)$ & $4(19)$ & $2(9.5)$ \\
\hline Sulfamethoxazole-trimethoprim (SXT) & $15(71.4)$ & 0 & $6(28.6)$ \\
\hline Tetracycline (TE) & $10(47.6)$ & $2(9.5)$ & $9(42.6)$ \\
\hline
\end{tabular}

TABLE 3: Antimicrobial resistance profiles of Salmonella isolates recovered from animal-origin food items.

\begin{tabular}{|c|c|c|c|c|c|}
\hline \multirow{2}{*}{ Antimicrobial tested } & \multicolumn{4}{|c|}{ Number of resistant isolates from food samples } & \multirow{2}{*}{ Total $(n=10)$} \\
\hline & Minced meat $(n=1)$ & Raw meat $(n=3)$ & Raw egg $(n=5)$ & Raw milk $(n=1)$ & \\
\hline Amoxicillin (AML) & $1(100)$ & 0 & $1(20)$ & $1(100)$ & $3(30)$ \\
\hline Ampicillin (AMP) & 0 & 0 & $1(20)$ & $1(100)$ & $2(20)$ \\
\hline Cephalothin (KF) & $1(100)$ & $1(33.3)$ & 0 & 0 & $2(20)$ \\
\hline Ceftriaxone (CRO) & 0 & 0 & 0 & 0 & 0 \\
\hline Gentamicin $(\mathrm{CN})$ & 0 & $1(33.3)$ & 0 & 0 & $1(10)$ \\
\hline Nalidixic acid (NA) & 0 & 0 & $2(40)$ & 0 & $2(20)$ \\
\hline Nitrofurantoin (F) & $1(100)$ & $1(33.3)$ & $2(40)$ & 0 & $4(40)$ \\
\hline $\begin{array}{l}\text { Sulfamethoxazole- } \\
\text { trimethoprim } \\
\text { (SXT) }\end{array}$ & $1(100)$ & $1(33.3)$ & $2(40)$ & 0 & $4(40)$ \\
\hline Tetracycline (TE) & $1(100)$ & $3(100)$ & $4(80)$ & $1(100)$ & $9(90)$ \\
\hline
\end{tabular}

nitrofurantoin. Looking at individual antimicrobials, resistance to tetracycline and sulfamethoxazole-trimethoprim was frequently observed, followed by ampicillin, amoxicillin, gentamicin, nalidixic acid, and nitrofurantoin. Two isolates (20\%) exhibited resistance to one antimicrobial, while eight $(80 \%)$ were resistant to two or more tested antimicrobials (Table 4). In general, antimicrobial susceptibility test revealed that ceftriaxone, gentamicin, and nalidixic acid were the antimicrobials indicated as active against Salmonella spp. isolated from this study.

\section{Discussion}

In the present study, the prevalence and the antimicrobial resistance patterns of Salmonella spp. isolated from foods of animal origin were evaluated. The study revealed an overall prevalence rate of 5.5\% (21 of 384) in the studied food items. Even though there have been some studies on Salmonella isolation in food items in Ethiopia [10, 12, 13], there has been no uniformity with respect to the sample type examined; as a result, the overall prevalence of Salmonella in animal-origin foods may not be comparable. In the combined analysis, the overall prevalence of Salmonella in this study was lower than that reported in Thailand [18] but higher than the reports in Morocco [19]. The difference might be the sample type and sample procedures and the detection methods employed for different studies. As Padungtod and Kaneene [20] described earlier, the rate of prevalence may also vary from experiment to experiment, from country to country, or from one area to another area within a country. There are many potential sources by which foods of animal origin can become contaminated; some are at the food preparation stage and others are in the food preparing environment. Some examples of contamination during food item processing and preparation are storage, washing, and cutting. As a public health view, most foodborne Salmonella infections are associated with foods of animal origin; however, recent investigations have identified fruits and vegetables as the source of contamination for foodborne Salmonella outbreaks [21]. Transport vehicles and processing equipment are also other examples of contamination. Unclean environment, unhygienic food handling practices, and water sources in public places are suggestive evidence of Salmonella postpreparation contamination in this study. Water of unknown microbiological quality can also be an important hazard for food items [21].

In the present study, Salmonella contamination in raw meat samples was $12 \%$, which was in close agreement with that of Ejeta et al. [13], who reported 12\% from retail raw meat samples in Addis Ababa, Ethiopia. In Ethiopia, approximately $15 \%$ of the meat samples were found to be positive at the retail level. But our finding was lower as compared to the studies conducted in Senegal [22] and in Iran [23]. However, the 
TABLE 4: Distribution of antimicrobial resistance among Salmonella spp. isolated from food items.

\begin{tabular}{lccc}
\hline Resistant pattern & Antimicrobial profiles & Resistant isolates & Source of food items \\
\hline Multidrug-resistant & AMP, F, SXT, TE & 1 & Minced meat \\
Multidrug-resistant & AML, AMP, NA, TE & 1 & Raw egg \\
Multidrug-resistant & KF, CN, SXT, TE & 1 & Raw meat \\
Multidrug-resistant & AML, AMP, TE & 1 & Raw milk \\
Multidrug-resistant & CN, SXT, TE & 1 & Raw egg \\
Multidrug-resistant & F, TE & 1 & Raw meat \\
Multidrug resistant & NA, SXT & 1 & Raw egg \\
Multidrug-resistant & SXT, TE & 1 & Raw egg \\
Monodrug-resistant & SXT & 1 & Raw egg \\
Monodrug-resistant & TE & 1 & Raw meat \\
\hline
\end{tabular}

Note. AML: amoxicillin; AMP: ampicillin; KF: cephalothin; CRO: ceftriaxone; CN: gentamicin; NA: nalidixic acid; F: nitrofurantoin; SXT: sulfamethoxazoletrimethoprim; TE: tetracycline.

prevalence of Salmonella in this study is greater than $5 \%$ in Thailand [24]. The variations in the prevalence could probably be due to improper handling of meat, personal hygiene of food handlers, improper cleaning of cutting utensils, and possible cross-contamination in butcher shops. Workers can play a significant role in spreading the contamination during retailing and preparation $[14,25]$. Recently, Garedew et al. [25] proved that butcher shop premises and utensils sanitation and personnel's hygiene were poor in this study area. There is a risk of Salmonella infection if the meat is improperly cooked and consumed raw, or if there is crosscontamination of Salmonella with other foods that are consumed raw [21]. The study also suggested that overall crosscontamination, environmental contamination, and food handler contamination might be considered as a common source of Salmonella along the food preparation. Salmonella infection can occur via cross-contamination of meat in slaughterhouses, butcher shops, and the kitchen during food preparation. Contaminated raw or undercooked red meats can serve as main vehicles of transmission for Salmonella [11, 25].

In our study, isolated Salmonella spp. in minced meat were $8 \%$, which was comparable with the previous studies done by Nyeleti et al. [10] (7.9\%) and Zewdu and Cornelius [26] (8\%) in Ethiopia. However, it was lower than that reported by Molla et al. [12] (12.1\%) and Ejeta et al. [13] (14.4\%). This prevalence difference might be due to variations of the methods used for traditional minced meat preparation for human consumption in the different geographical locations of the country. This is possibly due to the fact that minced meat is a combination of red meat parts from different sources that are eventually ground together [13], which could further magnify Salmonella contamination. This observation can be correlated to cross-contamination during handling and grinding of minced meat. In Ethiopia, traditionally prepared minced meat is often eaten raw or after minimal cooking, and it may be the potential source of Salmonella infection for the public in the country. In this study, only one burger sample was contaminated by Salmonella spp. The reason might be due to improperly cooked meat or poor personal hygiene of food handlers or inadequate cleaning of utensils and equipment or cross-contamination with other products like vegetables and fruits. Tomatoes have been indicated as a common food product involved in many Salmonella outbreaks [27]. On the other hand, an irregular water supply to most of the mass catering establishments in this study area made washing practices more difficult and, consequently, washing utensils and knives was infrequently practiced [25].

In the category of raw eggs, we recorded a contamination rate of $18 \%$. Various contamination levels were reported by numerous studies worldwide: $8 \%$ from chicken eggs in Dhaka city [28] and 24\% in Nigeria [29]. Conversely, the present finding was higher than $0.3 \%$ in Dhaka [30]. Salmonella prevalence from eggs in this study might be explained by environmental contamination during storage and transportation and ineffective cleaning of eggs. Moreover, the management system in practice could also be the probable reason for the variation of the prevalence. Egg contamination can occur in the infected ovary of the hen and also through egg contact with fecal material or it might be due to contamination of eggs during their supply from chicken owners to wholesale and retail markets. Salmonella detection from raw milk in the present study was comparable with a study conducted in Nigeria [31] and lower than 20\% prevalence in Ethiopia [32]. This difference might arise from milking contamination, unclean equipment, and poor hygiene of milk maid and handlers. However, it is higher than the prevalence of $2.1 \%$, which is found in Ethiopia [33]. As indicated by Garedew et al. [14], unclean environmental conditions and poor udder preparation might expose raw milk to bacterial contamination.

No Salmonella was isolated from pasteurized milk, cottage cheese, cream cake, egg sandwich, and pizza samples. Even though some studies reported Salmonella isolation from pasteurized milk in Ethiopia [26], proper pasteurization of the milk, the good sanitary condition of the processing plant, and absence of contamination after pasteurization of milk might be possible factors for negative samples. This finding is also supported by Garedew et al. [14], who explained that by the effectiveness of pasteurization at the processing plant we can minimize milk contamination to a lower level along the path from the cow to the pasteurization plant as well as 
during postpasteurization processing. In addition, the use of the right temperature for the storage of food items and ingredients used to prepare foods, clean cooking environment and equipment, and the good hygiene of storage and handlers are essential to reduce Salmonella contamination. The amount of Salmonella could have been too low to be detectable by cultural methods in this study.

In the present study, the antimicrobial-resistant Salmonella were found to be $47.6 \%$ of the total 21 Salmonella isolates tested, which is lower than those in previous studies conducted elsewhere in Ethiopia [25]. This observed resistance profile was higher than what other studies reported in Ethiopia (23.5\%) [26] and in Senegal (17\%) [22]. However, our findings were lower than $83 \%$ reported in Thailand [24]. Even though it needs a better understanding of antibiotics use in Ethiopia, this resistance variation might be due to indiscriminate use of antimicrobials in animal production without prescription in the animal health sector, which might favor selection pressure that increased the advantage of maintaining resistance genes in bacteria [34]. This is also supported by a high prevalence of Salmonella reported from humans and different food animals, with the strains having high levels of drug resistance $[35,36]$. Recently, the meta-analysis of animal Salmonella isolates resistant to drugs used against human salmonellosis in Ethiopia also showed that food animals are human exposure sources of antibiotic-resistant Salmonella [35].

In our study, most of the identified isolates are resistant to two or more antimicrobials, particularly to tetracycline (90\%), sulfamethoxazole-trimethoprim (40\%), nitrofurantoin (40\%), and amoxicillin (30\%). Salmonella resistance in this finding was higher than in the previous studies done in Ethiopia [26, 32] and other countries [22, 24]. The remarkable rise in the occurrence of antimicrobial resistance in Salmonella for the mentioned antibiotics was probably an indication of their frequent usage both in livestock and in public health sectors in Ethiopia. Studies conducted elsewhere in Ethiopia [35] have also indicated that the increase in the proportion of drug-resistant Salmonella isolates could be due to the irrational use of antimicrobials and inappropriateness of the prescription and dispensing methods in both the public veterinary and private health setups of the country. Due to the relatively limited access and high price to get the newly developed cephalosporin and quinolone drugs, the reports of prevalence of antimicrobial-resistant Salmonella to relatively low-priced and regularly available antibiotics are alarming for a low-income society living in most developing countries, like Ethiopia. However, it is important to note that these antibiotics are commonly used in veterinary medicine, and infections with these resistant Salmonella isolates could lower the efficiency of antibiotic treatment. The finding of this study shows slightly lower resistance than the study reported in Nigeria (93.1\%) [29]. In recent times, the frequency of antimicrobial drug resistance in Salmonella and the number of drugs to which the strains are resistant have increased worldwide, primarily as a consequence of antimicrobial use in food production. Recent reports have also highlighted the emergence of Salmonella with reduced susceptibility to fluoroquinolones and other drugs [9], which is associated with treatment failures and poor outcomes in human infections.

This study has limitations. We did not serotype identified Salmonella isolates for strain identification and characterization due to financial and lab facility constraints. In addition, we did not see the risk factors that might promote contamination and/or associations of Salmonella with foods of animal origin and drug resistance profiles. Therefore, this study's results should be considered as a baseline for bacteriological assessment of Salmonella isolates from foods of animal origin, which will be further serotyped and confirmed as foodborne pathogens by future studies in the same setting including food items of both animal and plant origin.

\section{Conclusion}

This study showed that Salmonella isolates were identified among the food items of animal origin, particularly for meat and its minced product, raw eggs, and raw milk. Salmonella drug resistance was also observed for almost all tested antibiotics that are commonly used in both veterinary and human health sectors in this study area and other parts of Ethiopia. This bacteriological assessment of Salmonella isolates from foods of animal origin can be used as a starting point to improve the city's foodborne pathogen surveillance system, as well as support hygiene and sanitation offices to assess and improve their public health and disease control programs. Therefore, our findings provide the prevalence and drug resistance of Salmonella from foods of animal origin and contribute information to scientists as well as public health researchers for the development of guidelines as well as food safety regulations to minimize the prevalent and resistant foodborne Salmonella species.

\section{Disclosure}

The authors are veterinary and public health professionals and members of the veterinary public health theme in subgroup of zoonosis and food safety research team at the University of Gondar. They are involved and interested in tuberculosis and foodborne zoonotic and food safety studies.

\section{Competing Interests}

The authors declare that they have no competing interests.

\section{Authors' Contributions}

Mebrat Ejo and Legesse Garedew conceived and designed the experiments. Zabishwork Alebachew, Walelgn Worku, and Legesse Garedew performed the experiments. Mebrat Ejo, Zabishwork Alebachew, and Legesse Garedew analyzed the data. Legesse Garedew and Walelgn Worku contributed reagents/materials/analysis tools. Mebrat Ejo and Legesse Garedew wrote the paper.

\section{Acknowledgments}

The authors would like to acknowledge the University of Gondar, Ethiopia, for the financial support of this study. 
They are also grateful to the staff members of the Faculty of Veterinary Medicine and the Institute of Public Health, University of Gondar, and Gondar Administration Bureau. In addition, they would like to appreciate the owners of the public catering establishments and personnel for their cooperation and participation in this study.

\section{References}

[1] R. S. Hendriksen, A. R. Vieira, S. Karlsmose et al., "Global monitoring of Salmonella serovar distribution from the world health organization global foodborne infections network country data bank: results of quality assured laboratories from 2001 to 2007," Foodborne Pathogens and Disease, vol. 8, no. 8, pp. 887-900, 2011.

[2] S. E. Majowicz, J. Musto, E. Scallan et al., "The global burden of nontyphoidal Salmonella gastroenteritis," Clinical Infectious Diseases, vol. 50, no. 6, pp. 882-889, 2010.

[3] F. Käferstein, "Foodborne diseases in developing countries: aetiology, epidemiology and strategies for prevention," International Journal of Environmental Health Research, vol. 13, no. 1, pp. S161-S168, 2003.

[4] F. M. Sánchez-Vargas, M. A. Abu-El-Haija, and O. G. GómezDuarte, "Salmonella infections: an update on epidemiology, management, and prevention," Travel Medicine and Infectious Disease, vol. 9, no. 6, pp. 263-277, 2011.

[5] E. Carrasco, A. Morales-Rueda, and R. M. García-Gimeno, "Cross-contamination and recontamination by Salmonella in foods: a review," Food Research International, vol. 45, no. 2, pp. 545-556, 2012.

[6] G. Tadesse, "Prevalence of human Salmonellosis in Ethiopia: a systematic review and meta-analysis," BMC Infectious Diseases, vol. 14, article no. 88, 2014.

[7] N. A. Feasey, G. Dougan, R. A. Kingsley, R. S. Heyderman, and M. A. Gordon, "Invasive non-typhoidal salmonella disease: an emerging and neglected tropical disease in Africa," The Lancet, vol. 379, no. 9835, pp. 2489-2499, 2012.

[8] WHO (World Health Organization), The Evolving Threat of Antimicrobial Resistance: Options for Action, WHO, Geneva, Switzerland, 2012.

[9] WHO (World Health Organization), Antimicrobial Resistance: Global Report on Surveillance, WHO, Geneva, Switzerland, 2014.

[10] C. Nyeleti, B. Molla, G. Hildebrandt, and J. Kleer, "The prevalence and distribution of Salmonellae in slaughter cattle, slaughterhouse personnel and minced beef in Addis Ababa (Ethiopia)," Bulletin of Animal Health and Production in Africa, vol. 48, pp. 19-24, 2000.

[11] L. Garedew-Kifelew, N. Wondafrash, and A. Feleke, "Identification of drug-resistant Salmonella from food handlers at the University of Gondar, Ethiopia," BMC Research Notes, vol. 7, article no. 545, 2014.

[12] B. Molla, D. Alemayehu, and W. Salah, "Sources and distribution of Salmonella serotypes isolated from food animals, slaughterhouse personnel and retail meat products in Ethiopia: 19972002," Ethiopian Journal of Health Development, vol. 17, no. 1, 2003.

[13] G. Ejeta, B. Molla, D. Alemayehu, and A. Muckle, "Salmonella serotypes isolated from minced meat beef, mutton and pork in Addis Ababa, Ethiopia," Revue de Medecine Veterinaire, vol. 155, no. 11, pp. 547-551, 2004.
[14] L. Garedew, A. Berhanu, D. Mengesha, and G. Tsegay, "Identification of gram-negative bacteria from critical control points of raw and pasteurized cow milk consumed at Gondar town and its suburbs, Ethiopia," BMC Public Health, vol. 12, no. 1, article 950, 2012.

[15] CSA (Central Statistical Authority), "The federal democratic republic of ethiopia, central statistical investigatory," Statistical Report, 2007.

[16] International Organization for Standardization (ISO) 6579, Microbiology of Food and Animal Feeding Stuff-Horizontal Method for the Detection of Salmonella, International Organization for Standardization (ISO), Geneva, Switzerland, 4th edition, 2002.

[17] CLSI (Clinical and Laboratory Standards Institute), Performance for Antimicrobial Disk Susceptibility Tests; Approved the Standard, CLSI Document M02-A11, CLSI, Wayne, Pa, USA, 11th edition, vol. 32, no. 1, pp. 1-76, 2012.

[18] S. M. Vindigni, A. Srijan, B. Wongstitwilairoong et al., "Prevalence of foodborne microorganisms in retail foods in Thailand," Foodborne Pathogens and Disease, vol. 4, no. 2, pp. 208-215, 2007.

[19] B. Bouchrif, B. Paglietti, M. Murgia et al., "Prevalence and antibiotic-resistance of Salmonella isolated from food in Morocco," Journal of Infection in Developing Countries, vol. 3, no. 1, pp. 35-40, 2009.

[20] P. Padungtod and J. B. Kaneene, "Salmonella in food animals and humans in northern Thailand," International Journal of Food Microbiology, vol. 108, no. 3, pp. 346-354, 2006.

[21] M. A. Gallegos-Robles, A. Morales-Loredo, G. Álvarez-Ojeda et al., "PCR detection and microbiological isolation of Salmonella spp. from fresh beef and cantaloupes," Journal of Food Science, vol. 74, no. 1, pp. M37-M40, 2009.

[22] A. Stevens, Y. Kaboré, J.-D. Perrier-Gros-Claude et al., "Prevalence and antibiotic-resistance of Salmonella isolated from beef sampled from the slaughterhouse and from retailers in Dakar (Senegal)," International Journal of Food Microbiology, vol. 110, no. 2, pp. 178-186, 2006.

[23] M. M. S. Dallal, M. P. Doyle, M. Rezadehbashi et al., "Prevalence and antimicrobial resistance profiles of Salmonella serotypes, Campylobacter and Yersinia spp. isolated from retail chicken and beef, Tehran, Iran," Food Control, vol. 21, no. 4, pp. 388-392, 2010.

[24] B. A. Gebre, "Qualitative screening of antibiotic residues and identification of antibiotic resistant Salmonella from raw and ready to eat meat in Thailand," International Journal of Advanced Life Sciences, vol. 5, pp. 51-64, 2012.

[25] L. Garedew, Z. Hagos, Z. Addis, R. Tesfaye, and B. Zegeye, "Prevalence and antimicrobial susceptibility patterns of Salmonella isolates in association with hygienic status from butcher shops in Gondar town, Ethiopia," Antimicrobial Resistance and Infection Control, vol. 4, no. 1, article 21, 2015.

[26] E. Zewdu and P. Cornelius, "Antimicrobial resistance pattern of Salmonella serotypes isolated from food items and personnel in Addis Ababa, Ethiopia," Tropical Animal Health and Production, vol. 41, no. 2, pp. 241-249, 2009.

[27] S. A. Bidol, E. R. Daly, R. E. Rickert et al., "Multistate outbreaks of Salmonella infections associated with raw tomatoes eaten in restaurants-United States, 2005-2006," Morbidity and Mortality Weekly Report, vol. 56, no. 35, pp. 909-911, 2007.

[28] M. M. Ahmed, M. M. Rahman, K. R. Mahbub, and M. Wahiduzzaman, "Characterization of Antibiotic Resistant Salmonella 
spp Isolated from Chicken Eggs of Dhaka City, Journal of Scientific Research, vol. 3, no. 1, pp. 191-196, 2010.

[29] E. O. Ekundayo and J. C. Ezeoke, "Prevalence and antibiotic sensitivity profile of Salmonella species in eggs and poultry farms in Umudike, Abia State," Journal of Animal and Veterinary Advances, vol. 10, pp. 206-209, 2011.

[30] K. Begum, T. A. Reza, M. Haque et al., "Isolation, identification and antibiotic resistance pattern of Salmonella Spp. from chicken eggs, intestine and environmental samples," Bangladesh Pharmaceutical Journal, vol. 13, no. 1, pp. 23-27, 2010.

[31] N. S. Karshima, V. A. Pam, S. I. Bata, P. A. Dung, and N. D. Paman, "Isolation of Salmonella species from milk and locally processed milk products traded for human consumption and associated risk factors in Kanam, Plateau State, Nigeria," Journal of Animal Production Advances, vol. 3, no. 3, pp. 69-74, 2013.

[32] T. Tadesse and A. Dabassa, "Prevalence and antimicrobial resistance of Salmonella isolated from raw milk samples collected from Kersa district, Jimma Zone, Southwest Ethiopia," Journal of Medical Sciences, vol. 12, no. 7, pp. 224-228, 2012.

[33] T. Liyuwork, T. Biruhalem, A. Sefinew, A. Haile, S. Zufan, and N. Haileleul, "Prevalence and antimicrobial resistance profile of Salmonella isolates from dairy products in Addis Ababa, Ethiopia," African Journal of Microbiology Research, vol. 7, no. 43, pp. 5046-5050, 2013.

[34] M. Gillings, Y. Boucher, M. Labbate et al., "The evolution of class 1 integrons and the rise of antibiotic resistance," Journal of Bacteriology, vol. 190, no. 14, pp. 5095-5100, 2008.

[35] G. Tadesse, "A meta-analysis of the proportion of animal Salmonella isolates resistant to drugs used against human salmonellosis in Ethiopia," BMC Infectious Diseases, vol. 15, no. 1, article 84, 2015.

[36] B. Molla, A. Berhanu, A. Muckle et al., "Multidrug resistance and distribution of Salmonella serovars in slaughtered pigs," Journal of Veterinary Medicine Series B: Infectious Diseases and Veterinary Public Health, vol. 53, no. 1, pp. 28-33, 2006. 

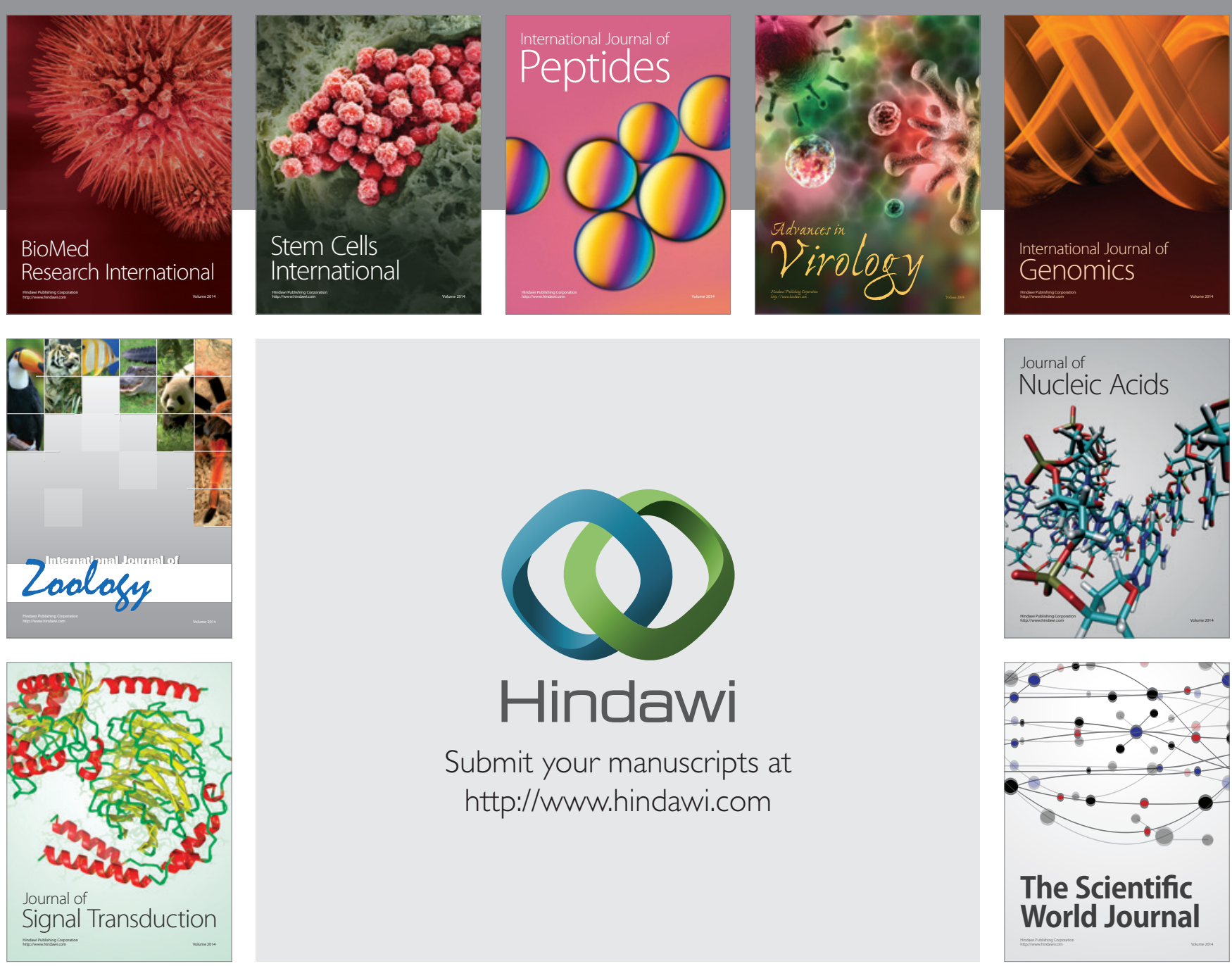

Submit your manuscripts at

http://www.hindawi.com
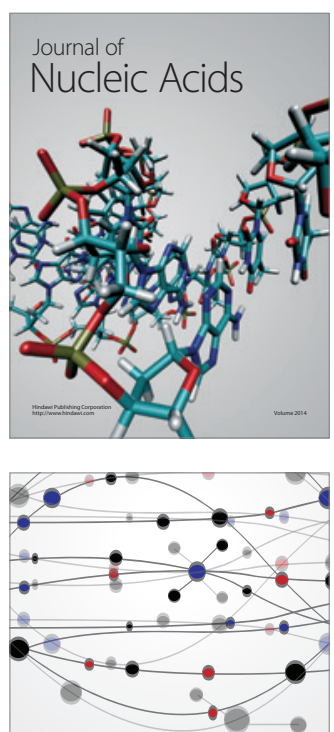

The Scientific World Journal
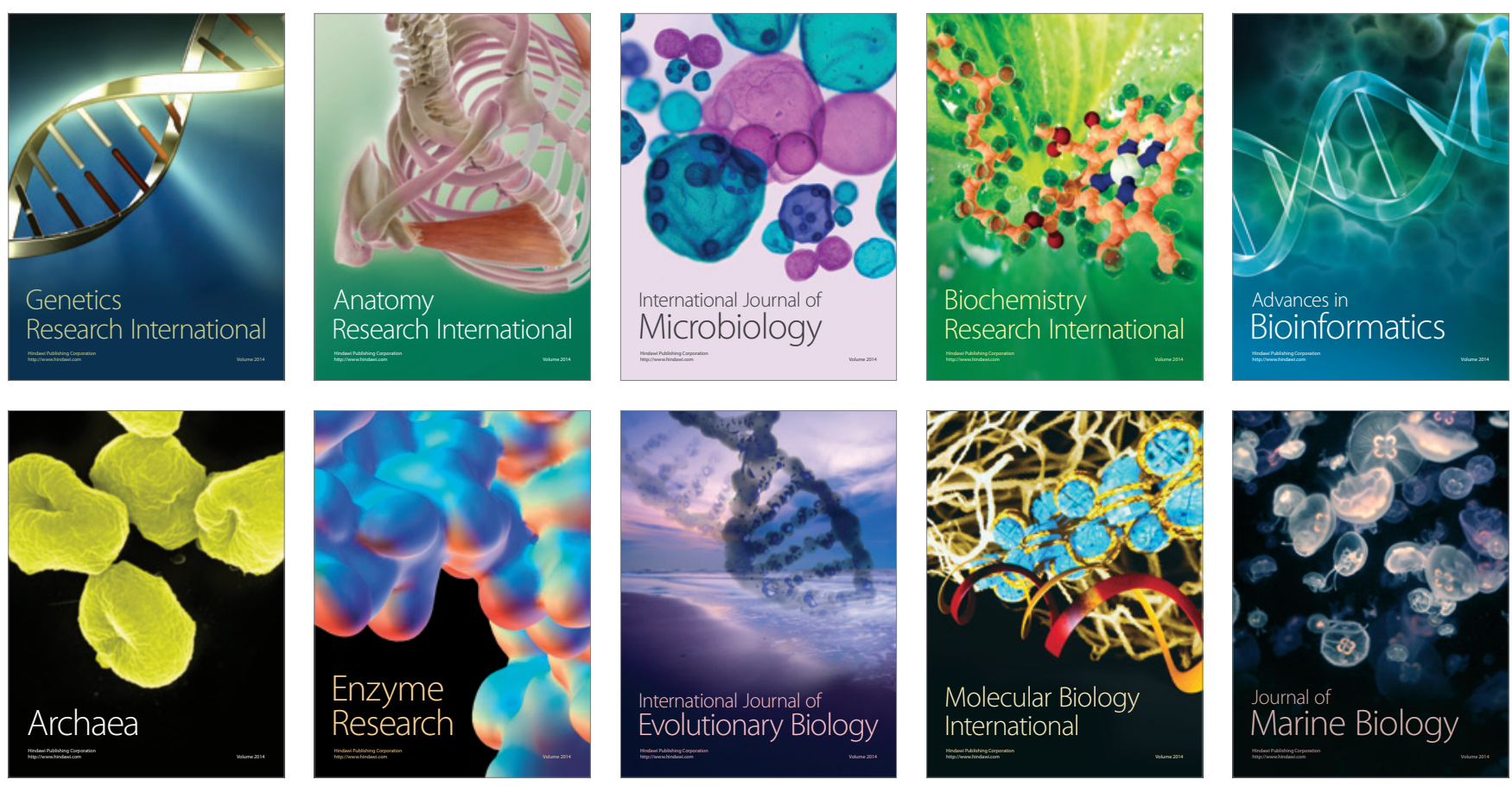